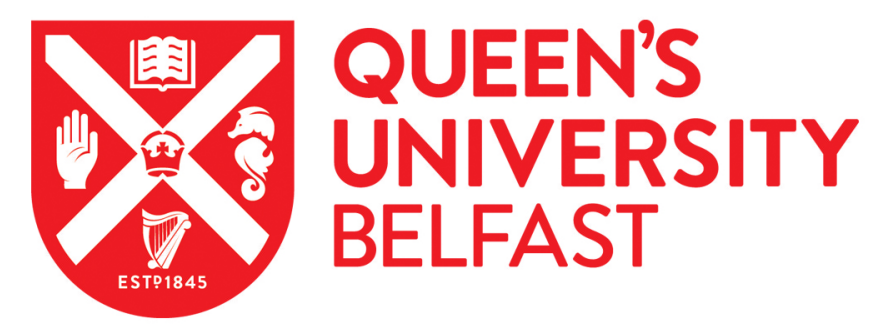

\title{
A spotlight on the management of complications associated with myeloproliferative neoplasms a clinician's perspective
}

Greenfield, G., \& McMullin, M. F. (2017). A spotlight on the management of complications associated with myeloproliferative neoplasms a clinician's perspective. EXPERT REVIEW OF HEMATOLOGY, 11(1), 25-35. https://doi.org/10.1080/17474086.2018.1410433

\author{
Published in: \\ EXPERT REVIEW OF HEMATOLOGY
}

Document Version:

Peer reviewed version

Queen's University Belfast - Research Portal:

Link to publication record in Queen's University Belfast Research Portal

Publisher rights

Copyright 2017 Taylor and Francis. This work is made available online in accordance with the publisher's policies. Please refer to any applicable terms of use of the publisher.

\section{General rights}

Copyright for the publications made accessible via the Queen's University Belfast Research Portal is retained by the author(s) and / or other copyright owners and it is a condition of accessing these publications that users recognise and abide by the legal requirements associated with these rights.

Take down policy

The Research Portal is Queen's institutional repository that provides access to Queen's research output. Every effort has been made to ensure that content in the Research Portal does not infringe any person's rights, or applicable UK laws. If you discover content in the Research Portal that you believe breaches copyright or violates any law, please contact openaccess@qub.ac.uk. 
A spotlight on the management of complications associated with Myeloproliferative Neoplasms: A clinician's perspective

G. Greenfield, M.F. McMullin

Queen's University, Belfast, N. Ireland

m.mcmullin@qub.ac.uk 


\section{A spotlight on the management of complications associated with Myeloproliferative Neoplasms: A clinician's perspective}

\section{Summary}

Myeloproliferative neoplasms are associated with a variety of symptoms and signs which cause major morbidity for the patients. The disorders are associated with increased incidence of thromboembolic and hemorrhagic events which can lead to complications and shortened life expectancy. Using systematic literature review and expert clinical and research experience the authors discuss strategies for the management of symptoms and signs including pruritus, fatigue, splenomegaly and cytopenia. Cytoreduction including treatments to inhibit the JAK/STAT pathway are considered. Pathogenesis and prevention and treatment of thrombotic and hemorrhagic events and their management is addressed and the suggested management of the special situations such as surgery and pregnancy are discussed. Likely developments in the field are explored in the expert commentary and possible advances in the next 5 years.

\section{Keywords}

Myeloproliferative neoplasms, fatigue, pruritus, thromboembolic events, surgery, hemorrhage, hydroxycarbamide, interferon, ruxolitinib

\subsection{Introduction}

It is over 60 years since Damashek described the links between high blood counts and potential disease phenotypes [1] as myeloproliferative diseases. These have been defined and classifications refined with the most recent version from the World Health Organisation (WHO) published very recently [2]. This provides diagnostic criteria for the three main types of myeloproliferative neoplasms (MPN), polycythemia vera (PV, essential thrombocythemia (ET) and myelofibrosis (MF) which can be primary or secondary to PV or ET.

The MPNs are acquired clonal disorders. Mutations in the genes JAK2, MPL or CALR are commonly found and indicate the presence of an acquired clone [3-5]. In these disorders, the pathway downstream of the mutated protein is upregulated and this activated cytokine pathway can lead to symptomatology.

Patients with MPN have increased complications. High blood counts are associated with thromboembolic and haemorrhagic events. Special situations such as pregnancy or surgery are therefore of increased risk and need to be managed. The diseases and their treatments can lead to cytopenias particularly anemia which may require specific management.

Symptoms and complications associated with the MPNs and available management options will be discussed.

\subsection{Symptoms in MPN}

The disease burden in MPN leads to a variety of symptoms which can be very troubling to patients and can lead to premature mortality. The need to document and measure symptom burden in these patients both in trials and in evaluating patient responses in general has led to the development of a number of tools to evaluate symptoms. The Myelofibrosis Symptom Assessment Form (MF-SAF) is a 20-item scoring system designed and validated to measure symptoms in myelofibrosis [6]. This was then expanded to capture symptoms in ET, PV and MF in the Myeloproliferative Symptom 
Assessment Form (MPN-SAF) which was useful in evaluating the symptom burden in the various disease types and has been validated in a number of languages [7]. This was consolidated as a 10item questionnaire which can be rapidly administered in a clinical environment, the MPN-SAF Total Symptom Score (TSS) [8].

Of note in symptom assessment, the MPN-SAF was administered in the MOSAICC (Myeloproliferative Neoplasms: An in -depth Case- Control) study to both patients with ET, PV and MF and population controls. MPN patients had significantly higher scores than the controls for 25 out of the 26 symptoms measures indicating that quality of life was significantly worse in MPN patients compared to controls [9].

A wide variety of symptoms have been reported and assessed using these and other instruments. Self-reporting in large cohorts of patients also record a variety of symptoms with fatigue the most common followed by pruritus, night sweats, bone pain, fever and weight loss [10]. Some of the most burdensome and prevalent are discussed further with any suggestions for management.

\subsection{Fatigue}

Patients with a variety of disorders often complain bitterly of fatigue which is profoundly difficult for physicians to evaluate. However, it is the most common self-reported symptom in patients with MPNs [10]. The fatigue is reported to have a major influence on quality of life and ability to carry out the daily activities of living. Whilst anemia may be a contributory factor, it certainly does not explain the fatigue in many MPN patients who manifest it in early stages before there are any cytopenias. As with many of the symptoms in MPN the cytokine storm due to activation of the JAK/ STAT pathway may be a factor. Deconditioning, where the fatigued patient restricts their activity may also worsen the situation.

Specific treatments for the MPN may help by interfering with the dysregulated JAK/STAT pathway. Otherwise there is ongoing debate as to whether graded controlled exercise helps the situation and protects the patient. Some find this helpful while other patients have advocated that vigorous training as beneficial. A recent small study of a 12 week intervention with Yoga showed improvement in symptoms including fatigue[11] so possibilities for non- pharmacological intervention in fatigue may be considered

\subsection{Pruritus}

Pruritus is a prevalent symptom in many MPNs. It can be extremely disabling, interfering with sleep and has led to suicidal intent in some cases. Severe aquagenic pruritus can be a major factor which interferes with many activities of daily living. This, almost pathognomonic symptom of MPN, has a poorly understood aetiology. Increased histamine and its release has long been thought to be associated [12]. Basophils have been found to be increased, hypersensitive, constitutively activated associated particularly in those with a JAK2 clone as part of an activated JAK/STAT pathway [13]. Histamine release and the activated basophils are thought to contribute to the aetiology of the pruritus.

Empirical therapies have been tried for this very disabling problem. Antihistamines are a logical choice and there are early reports of relief of symptoms with cyproheptadine [12]. The availability of histamine $\mathrm{H} 2$ receptor antagonists, allowed them to be assessed and they were found to led to improvements in pruritus in $44 \%$ of patients on cimetidine in a study [14]. Reports of idiopathic pruritus improving with selective serotonin reuptake inhibitors (SSRI) led to a small trial of treatment of 5 patients with paroxetine. All experienced prompt and marked improvement of symptoms so this 
is another therapy worthy of consideration [15]. Further reports in small series of patients report very rapid and favourable responses to SSRIs [16].

There are reports of phototherapy as effective therapy in controlling PV associated pruritus $[17,18]$. Oral psoralen phototherapy (PUVA) was found to be effective in a series of 5 patients with aquagenic pruritus including those with PV but relapse occurred weeks after discontinuation of therapy [19]. Broadband ultraviolet (UV) B phototherapy has been reported as being efficacious [20]. Narrowband UVB was also shown to be effective in the treatment of aquagenic pruritus in a series of 10 patients. Complete remissions occurred within 2 to 10 weeks of treatment in 8 of 10 patients [21].

Thus, there are a number of empirical therapies which have been reported to be effective for pruritus in small numbers of patients. The other approach to pruritus is the effect of specific therapies used for cytoreduction. Interferon alfa has been reported in some series to improve intractable pruritus $[22,23]$. Treatment with other cytoreductive therapies produce very variable and inconsistent responses.

The JAK/STAT pathway inhibitor ruxolitinib was first introduced for the treatment of the MPN myelofibrosis and, as well as other effects, major responses in pruritus are seen. Over $50 \%$ of patients had an improvement of $50 \%$ or more in symptoms like pruritus [24]. This was also seen when this drug was used in trials in PV with major improvements in pruritus measured in addition by specific pruritus symptom impact scale while on the drug [25]. These major and life changing improvements in pruritus symptomatology is a common observation in clinical practice using JAK inhibitors. This is thought to be due to inactivation of cytokines in the constitutively activated JAK/STAT pathway.

\subsection{Constitutional symptoms and other symptoms}

There are a number of other symptoms which have been measured and are specifically associated with some or all of the MPNs. In MF in particular there is a hypercatabolic state so that patients describe that they are eating well but lose weight. The hypercatabolic state may contribute to symptoms such as fevers and night sweats. Some also have a lot of abdominal problems.

Splenomegaly can cause major symptomatology in patients particularly with lead to feeling of fullness and early satiety. Splenic infarcts can lead to abdominal pain.

MPNs cause and contribute to vascular events. Microvascular pathology can lead to a variety of related symptoms such as headaches, concentration problems, dizziness and sexual-related complaints [26].

\subsection{Symptom management options}

There are no particular treatments for any of the other symptoms. However, cytoreductive treatments in reducing the blood counts and perhaps reducing spleen size in those with splenomegaly may improve symptoms. Interferon has been described as helping particularly pruritus [23] but it may improve other symptoms. However, it can be poorly tolerated and thus cause more symptoms. Hydroxycarbamide/hydroxyurea (HU) will reduce splenomegaly and blood counts and may bring about some improvements in wellbeing although frequently patients continue to complain of tiredness and other problems despite improvement in blood parameters.

The JAK inhibitors particularly ruxolitinib which is now first line treatment for PMF and second line for PV have been shown to be particularly efficacious in reducing the spleen size and reducing 
symptoms and bring about improvement in the overall patient condition. This have been a major advance in the management of patients with MPN $[24,25]$.

Prior to JAK/STAT inhibitors splenectomy could be considered for particularly troublesome splenomegaly either because of size and pain or in those where cytopenias particularly with high transfusion requirements was an issue. There was some consideration that splenectomy might be associated with blast transformation[27], however it may still be a possible therapeutic option for those with spleen related mechanical symptoms refractory to ruxolitinib[28]. Splenic irradiation has also been used as a palliative measure in those with a troublesome spleen. Effects are transient and worsening cytopenia may be an issue but it is something to consider[29].

\subsection{Thromboembolic complications}

Both arterial and venous thromboembolic events are common complications of each of the myeloproliferative neoplasms. They contribute significantly to morbidity associated with the disorders and are estimated to contribute to around $40 \%$ of mortality [30,31]. Treatment and prevention of these common complications form a key part of good quality care.

\subsection{Thrombosis in Polycythemia Vera}

Despite being a recognised disease entity for a significant time, the exact natural history is poorly defined and most major observational studies are confounded by varying treatment modalities. One observational study of 1213 PV patients showed a rate of fatal or non-fatal thrombosis of approximately $40 \%$ either before presentation, at presentation or during follow-up. The Incidence of thrombosis was 3.4\% per year [30]. A more recent retrospective cohort of 1545 patients with PV recorded pre-diagnosis arterial and venous thrombotic events in $16 \%$ and $7.4 \%$ of the cohort respectively. A significant association with arterial events during follow-up was demonstrated between hypertension and prior arterial thrombus whilst a prior venous thrombosis was significantly associated with a further venous event [32].

The observational arm of the European Collaboration of Low dose Aspirin in Polycythemia (ECLAP) trial followed 1638 patients for a mean duration of 2.7 years. Of these patients, $36 \%$ had a prior history of thrombosis at or before diagnosis with a high proportion occurring in the 2 years prior to diagnosis $[31,33]$. This points to a latent period of the MPN during which the thrombotic risk is increased prior to the full phenotypic presentation becoming evident. During follow-up 205 further thrombotic events were recorded in 169 patients with approximately $70 \%$ arterial events. Only $40 \%$ of these events were first time thrombotic events. Older age and prior history of thrombosis were identified as the key predictors of arterial or venous thrombosis [31,33]. It was observed that a history of myocardial infarction of cerebrovascular event predicted recurrence of the same whilst prior venous thrombosis was associated with further venous events without increasing the risk for arterial events. Increased haematocrit ( $\mathrm{Hct}$ ) and platelet count were not associated with thrombotic risk but a total leucocytosis of greater than $15 \times 10^{9} / \mathrm{L}$ was associated. Hypertension showed a mild non-significant association whilst smoking was strongly associated with risk of thrombosis. [31] The overall risk of cardiovascular mortality was 1.4 times that of appropriate age and sex matched individuals at that time [33].

\subsection{Thrombosis in Essential Thrombocythemia}


In ET, the risk of thrombotic events is lower than is seen in PV. The PT-1 trial which compared anagrelide and aspirin against $\mathrm{HU}$ and aspirin demonstrated an overall rate of arterial and venous thrombosis of approximately $13 \%$ and $8 \%$ in each arm respectively during a median follow-up of 39 months [34]. The genetic landscape is also more varied with regards to driver mutations. JAK2V617F mutations present in around $50 \%$ of ET patients have been clearly shown to be an independent risk factor for thrombosis [35] and in some data sets JAK2 mutated ET has the same risk of thromboisis as PV[36]Despite the higher platelet count seen in CALR positive ET, the presence of this mutation confers a lower risk of thrombosis [37].

The relative scarcity of the MPL mutation, occurring in approximately $3-15 \%$ of ET patients results in few patients with this genetic background having thrombotic events. The PT-1 trial did not identify MPL mutation as a risk factor for thrombosis. [38] This suggests that the mutated JAK2V617F plays a significant role in the pathogenesis of thrombus formation.

\subsection{Thrombosis in Myelofibrosis}

The rate of thrombotic events in MF is less well established due to a higher rate of competing complications such as cytopenias, infection and leukemia transformation which contribute significantly to mortality and morbidity. There is also some phenotypic variation depending on whether the aetiology is primary or a secondary transformation from PV or ET. A recent analysis has suggested that the rate of thromboembolic events is similar to that seen in ET and lower than that seen in PV. Similar to ET, there is also a demonstrated higher rate of thrombosis in the presence of $J A K 2$ V617F mutation in comparison to patients with a CALR, MPL mutation or "triple negative" genotype. [39] The benefit in thrombosis risk in triple negative PMF is offset by the higher risk of leukemic transformation in this group.

The recently defined early/prefibrotic myelofibrosis which is differentiated from ET and overt PMF on the basis of bone marrow biopsy findings appears to have a higher rate of thrombosis compared to ET highlighting the importance of the bone marrow biopsy as part of the diagnostic work-up. [40]

In clinical practice PV and ET patients are classified as high risk of thrombosis on the basis of the presence of one or both of age greater than 60 years and/or history of previous thrombosis. [41,42]

\subsection{Venous Thrombosis in Unusual Sites}

It has also become evident that MPNs are associated with venous thrombosis at unusual sites. Splanchnic vein thrombosis (SVT) describes a heterogeneous group of thrombotic pathology occurring within the hepatic veins (Budd Chiari Syndrome) (BCS), portal vein, splenic vein, mesenteric vein or combination of the above. There is a strong proven association between SVT and underlying MPN as a causal factor. In a meta-analysis it was demonstrated that $40.9 \%$ of SVT and $41.1 \%$ of BCS have an underlying MPN. They also demonstrated $41.1 \%$ of PVT and $27.7 \%$ of BCS patients have the JAK2V617F mutation. [43] CALR mutations have also been demonstrated in small numbers of JAK2V617F negative SVT patients with MPN. [44,] Therefore SVT is widely considered a presenting complication of MPN disease. The prevalence of MPN in SVT is significantly higher than in any other venous thrombosis.

The consensus in the published guidelines has agreed that individuals presenting with an unexplained SVT should undergo a diagnostic workup for an underlying MPN [45]. These patients 
often do not display the classical phenotypic blood counts expected in MPN. Therefore, the absence of polycythemia, leucocytosis or thrombocytosis should not exclude appropriate testing in SVT where there is no local provoking factor. The lack of these characteristic findings may be due to hypersplenism, variceal bleeding and/or associated iron deficiency. Given this phenotypic diversity many of the patients do not fit neatly into one classification of MPN but rather are diagnosed with an MPN unclassifiable.

It has been suggested that a small number of $J A K 2 \mathrm{~V} 617 \mathrm{~F}$ negative patients may develop positivity over subsequent months following SVT. These patients appear to fall into a higher risk group for thrombotic complications. [46] A positive MPN diagnosis has been suggested as an independent risk factor for mortality following SVT [47]. Although unlikely to play any role in the clinical setting the presence of the JAK2 46/1 haplotype has been described to confer susceptibility to JAK2V617F positive MPNs presenting with SVT [48].

\subsection{Mechanisms of Thromboembolism}

There is a multi-factorial disruption of normal hemostatic mechanisms which produce a procoagulant state in MPN. Contributing to this is an abnormal interplay between erythrocytes, leucocytes, platelets, endothelial cells and plasma proteins alongside the quantitative effect of increased cell number and a qualitative effect of abnormal clonal cell membrane proteins and interactions.

In PV the effect of increased Hct results in an increased plasma viscosity. This distorts the flow characteristics of the blood, a key feature of Virchow's triad resulting in a higher rate of thrombosis particularly in low shear venous beds. The increased red cell mass may displace platelets towards endothelial cells causing an increased rate of interaction and activation. Leucocytosis has been identified in both ECLAP and CYTO-PV studies as a significant risk factor for thrombosis in PV. [31,49] Leucocytosis has also been consistently identified in ET as a risk factor for thrombosis in multi-centre trials $[50,51]$. The IPSET prognostic scoring system a leucocytocyte count greater than $11 \times 10^{9} / \mathrm{I}$ as a part of the predicitve model[52].

Changes to the cells themselves may be of even greater significance than simply an increased number of cells. PV red cells show an increase in binding to endothelial laminin as a result of an JAK2V617F driven activation of Lu/BCAM [53]. Platelets and leucocytes show phenotypic changes associated with activation. Demonstrating increased thromboxane $A 2$ metabolites, a key feature of platelet activation, in the urine of MPN patients was part of the basis for the ECLAP trial assessing the effect of aspirin in reducing thrombosis risk [54]. Increased levels of platelet activation products in the plasma have also been demonstrated [55].

Expression of P-Selectin and Tissue Factor on platelets and leukocyte CD11b expression is higher in MPN patients. The presence of JAK2V617F mutation is associated with a higher extent of platelet activation [56] Activated leucocytes and platelets have key interactions and higher levels of leucocyte platelet aggregates are present in MPN patients [57].

The increased rate of thrombosis in JAK2V617F positive ET and PMF patients in comparison to patients with an alternative driver mutation suggests a direct role of the JAK2V617F mutation in the pathophysiology of thrombus. Although 95\% of PV patients harbour the JAK2V617F mutation, there is a variation in the allele burden present. There is conflicting evidence to support an association between higher allele burden and thrombosis risk [39]. 
Systemic inflammatory conditions exhibit a higher rate of thrombosis. In MPN there is increased secretion of pro-inflammatory cytokines including IL-1, IL-2, IL-8, TNF- $\alpha$ and IFN- $\gamma$ and reduced secretion of inhibitory cytokines including IL- 4 and IL-10. There is also a positive association with higher levels of growth factor production including granulocyte-macrophage colony-stimulating factor (GM-CSF), platelet-derived growth factor (PDGF) and vascular endothelial growth factor (VGEF) evident in MPN [58]. In the general population there is an association between increasing Creactive protein (CRP) and cardiovascular risk. Indeed the use of monoclonal antibody therapy targeted against IL-1 $\beta$ cytokine reduced risk in the general population [59]. In MPN, a recent study of 244 patients with ET and PV showed a higher risk of thrombosis positively correlated with higher plasma high sensitivity CRP and pentraxin-3 (PTX-3) levels [60]. Exploring the role of inflammation modification in MPN may yield potential new therapeutic options.

The role of traditional cardiovascular risk factors must also be also be reviewed. More recent cohort studies of PV patients have shown lower rates of thrombosis in comparison to the initial ECLAP trial. It is notable that in each case the management of modifiable cardiovascular risk factors has been more aggressive [61]. The IPSET-Thrombosis prognostic scoring system identified cardiovascular risk factors (hypertension, smoking and diabetes) as independent predictors of thrombosis in ET [35].(See Table)

The influence of clones in atherosclerosis has recently been been noted where clonal hematopoiesis of indeterminate origin (CHIP) which include clones with mutations in TET2 and JAK2 were associated with a 1.9 times as great a risk onf coronary artery disease compared to those without clones[62].

\subsection{Management of thrombosis}

From the perspective of the clinician it is therefore key to institute therapy with the goal of preventing initial or recurrent thrombotic events in MPN. Treatments have been guided by a number of large multi-centre randomized control trials undertaken over the last two decades. The strategies available to the clinician include the use of anti-platelet drugs, cytoreduction by venesection or pharmacological means, targeted JAK2 inhibition, anticoagulation and modification of existing cardiovascular risk factors.

\subsection{Antiplatelet agents}

The ECLAP trial recruited 518 patients with PV to a randomized, double blinded, placebo controlled study, examining the role of aspirin in the prevention of thrombosis. Multi-variate analysis which adjusted for smoking and additional cardiovascular risk factors confirmed a significant reduction in the combined primary end points of fatal and non-fatal arterial and venous thrombosis. This was reported as a relative risk reduction of $60 \%$ for non-fatal myocardial infarction, non-fatal stroke, pulmonary embolus, major venous thrombosis or death from cardiovascular cause [54]. Therefore, where there is no contraindication to aspirin therapy this should be initiated in PV patients. Current guidance would suggest that this applies to patients in both the high and low risk groups. [41,,63] Where aspirin is contra-indicated it may be reasonable to consider the use of an alternative antiplatelet agent.

There is little high quality evidence for the risk versus benefits of anti-platelet therapy in ET. Systematic review evaluated the modest risk reduction of thrombosis risk with anti-platelet therapy with significant bleeding risk[64].The role for aspirin in the management of ET has been inferred 
from the ECLAP study in PV. Aspirin has been clearly demonstrated to show a reduction in the rate of thrombosis in JAK2V617F positive ET patients. However, aspirin did not reduce the risk of thrombosis in low risk CALR positive patients but led to an increased bleed risk [65]. Therefore, it is reasonable to consider the omission of aspirin in $J A K 2 \mathrm{~V} 617 \mathrm{~F}$ negative low risk ET patients without any additional cardiovascular risk factors. As discussed below, extreme thrombocytosis is an independent risk for haemorrhage and therefore aspirin use when the platelet count is greater than $1500 \times 10^{9} / \mathrm{L}$ should be generally be avoided.

\subsection{Cytoreduction}

Hct control in PV was examined in the CYTO-PV study. A total of 326 adults were recruited with a target Hct of less than $45 \%$ for one arm and a target of $45-50 \%$ for the other. Venesection, the use of $\mathrm{HU}$ or both were acceptable for Hct reduction. All patients received low dose aspirin. The primary end point of time to death from cardiovascular cause or major thrombosis showed a significant benefit from tighter Hct control, reducing the rate from 4.4 to 1.1 events per 100 person years with a target of less than $45 \%$. Total cardiovascular events were also significantly reduced in the lower Hct arm and no statistically significant difference in bleeding was noted [66].

Venesection is a useful first line therapy with which Hct control can be achieved. Current guidelines recommend its use in both low and high risk patients with a target Hct of less than $45 \%$.

Cytoreductive agents should be reserved for those in the high risk category. The European Leukemia Net guidelines in 2011 recommended the use of HU or IFN- $\alpha$ as first line agents and this is confirmed in the recent European Society of Medical Oncology guidelines [41, 67].

$\mathrm{HU}$ is an effective agent in reducing cell counts and is generally well tolerated with few side effects. There is no randomized controlled trial data which demonstrates a categorical reduction in thrombotic events on this drug. However, retrospective analysis of the ECLAP observational study allowed selection of 1042 patients who received either venesection alone or HU to maintain a haematocrit of less than $45 \%$. Propensity scoring demonstrated a significantly reduced rate of fatal and non-fatal cardiovascular events from 5.8 to 3.0 per 100 person years. The difference was restricted to high risk patients therefore providing some evidence to back up current guidelines [68].

There is some debate regarding the propensity of HU treated patients towards leukemic transformation. Given the natural history of transformation and the confounding nature of other cytoreductive therapies that patients may have previously been exposed to, there has never been any conclusive proof that $\mathrm{HU}$ increases the risk of leukemia [69].

In ET the role of HU was examined in the PT-1, ANAHYDRET and EXELS studies. ANAHYDRET demonstrated non-inferiority of anagrelide in comparison to HU [70]. The other two studies demonstrated a reduction in arterial thrombosis rate but an increase in venous thrombosis in patients treated with $\mathrm{HU}$ in comparison to patients treated with anagrelide [34, 71]. Anagrelide works specifically to reduce platelet counts by inhibiting megakaryocyte maturation. It has an equivalent effect to $\mathrm{HU}$ in reduction of the platelet count and is recommended as an option in ET where patients have failed on or been intolerant of $\mathrm{HU}[41,42,67]$.

Interferon Alpha therapy is an additional effective agent for cytoreductive therapy. Pegylated formulations of the drug appear to have better tolerability than the conventional formulations. Although there are no published data on the ability of these agents to reduce the rate of thrombosis it has been shown that they can bring about hematological remission and a reduction in the JAK2V617F allele burden $[72,73]$. This response may be dependent on the presence of additional driver mutations such as TET 2 which appears to negatively affect the rate of complete molecular 
remission. The more widespread availability of genomic sequencing alongside further studies into the response to these agents may help to identify sub groups of patients who would gain the most benefit. In current guidance Interferon alpha finds a role in the younger patient as a first line cytoreduction agent as this agent is deemed to be non leukemogenic [41, 42, 63].

With extreme thrombocytosis in an emergency situation thrombocytopheresis could be considered. In most situations urgent cytoreductive therapies are what is needed but there may be individual cases where thrombocytophersis should be considered as a possible therapeutic option [74].

\subsection{JAK inhibition}

The impact of JAK inhibition to reduce the rate of thrombosis independent of other factors has not yet been categorically demonstrated. The RESPONSE trial compared Ruxolitinib to best available therapy in PV patients resistant to or intolerant of HU. Patients all took low dose aspirin and had mandatory venesection defined by Hct readings. The rate of thromboembolic events in the ruxoloitinib arm was lower than expected in this high-risk population [25].

Ruxolitinib has demonstrated a small but significant reduction in JAK2 V617F allele burden in PV/ET and MF patients [75,76,77]]. As for Interferon alpha therapy, whether this reduction in allele burden correlates to a reduction in thrombosis risk is as yet unclear. This is important to establish going forward.

\subsection{Other Modifiable Risk Factors}

The role of conventional modifiable cardiovascular risk factors in the general population must also be addressed in the MPN population. All relevant guidelines highlight the role of aggressive management of conventional cardiovascular risk factors $[41,42,63,67]$. Smoking cessation advise and support through relevant local services are essential. Good diabetic control, lipid monitoring and appropriate use of lipid lowering therapy and blood pressure control will all form part of the holistic care of the MPN patient.

There is also recent interest in the role of ACE inhibitors in the prevention of erythrocytosis. They have a defined role in the management of post renal transplant erythrocytosis [77]. A retrospective analysis of the ECLAP observational cohort revealed that patients using ACE inhibitors were treated significantly less frequently with chemotherapy in comparison to those treated with alternative antihypertensives [61]. Prospective studies looking into rates of thrombosis, alongside the requirement for therapy would be required before advise to preferentially prescribe ACE inhibitors for blood pressure control could be made.

\subsection{Anticoagulation}

Where venous thromboembolism occurs there is a clear indication for anticoagulation rather than anti-platelet medication in the MPN population as for the general population. Anti-platelet medication used concurrently with anti-coagulation is associated with a significantly elevated bleed risk and therefore should only be considered with caution. Atrial fibrillation (AF) should also be anticoagulated in line with current guidelines.

Traditionally low molecular weight heparin and oral vitamin $\mathrm{K}$ antagonists have been used for the purposes of anticoagulation Within the last decade the arrival of factor Xa and direct thrombin inhibitors classed together as novel oral anticoagulants (NOACs) have made a major impact on anticoagulation within the general population. NOACs have demonstrated non-inferiority to warfarin for stroke prophylaxis in AF and in the treatment of venous thromboembolic events $[79,80]$. These 
agents have not been evaluated prospectively in the setting of MPN and these medications are not routinely used in the prevention or treatment of VTE in other malignant neoplastic disorders. A small observational study has suggested that the rate of hemorrhagic events in 25 MPN patients treated with NOACs for either VTE or stroke prophylaxis in AF does not differ significantly from a case control population treated with Aspirin [81]. A prospective trial demonstrating non-inferiority to current therapeutic strategy is required to recommend routine prescribing.

Opinions on the management of thrombosis in MPN patients are heterogeneous[82], However, currently, there are no true disease modifying agents and therefore as the acquired clone remains MPN must be considered a permanent risk factor for thrombosis and thus anticoagulation should be continued indefinitely unless risk outweighs benefit. SVT often presents unique challenges with regards to anticoagulation and is discussed in more detail below.

\subsection{Hemorrhage}

Hemorrhagic complications of MPNs are not infrequent. They range from minor bleeding events to major fatal hemorrhage. A recent prospective observational study of German MPN patients showed that in contrast to the peak of thrombotic events occurring at the time of MPN diagnosis, the majority of hemorrhagic events following diagnosis. They suggest this demonstrates that hemorrhage is due to complications of the MPN itself or treatment strategies [83]. There are a number of aetiologies of hemorrhage that have been defined.

There is a paradoxical increased risk of haemorrhage associated with extremes of thrombocytosis. Where the platelet count is above $1000 \times 10^{9} /$ there is a reduction in the quantity of circulating large multimers of von Willebrand's factor due to binding on the platelet surface. This results in an acquired von Willebrand's disease [84]. Use of aspirin or other anti-platelet medications will then further disrupt normal clot formation and can result in significant haemorrhage. Aspirin is commonly avoided when the platelet count exceeds $1500 \times 10^{9} / \mathrm{I}$. A platelet count between 1000 and $1500 \mathrm{x}$ $10^{9} /$ I should prompt consideration of von Willebrand factor antigen level and risocetin co-factor activity to exclude acquired von Willebrand's disease prior to initiation of aspirin therapy [85].

In PMF disruption of normal hematopoiesis can lead to thrombocytopenia which at extreme levels pre-disposes to spontaneous hemorrhage and exaggerates traumatic hemorrhage. Platelet transfusion may be required as prophylaxis at extremes of thrombocytopenia or as a therapeutic intervention during hemorrhage. Disease related splenomegaly and cytoreductive therapies may also cause thrombocytopenia.

The use of anti-platelet agents as therapeutic strategies for the prevention of thromboembolic events has a risk of hemorrhage. Careful attention to the platelet count is required. In the general population a recent prospective cohort study looking at the effect of aspirin on the fatal and nonfatal bleeding in patients receiving the drug for secondary prevention of vascular events showed an increasing annual rate of major haemorrhage from $1.5 \%$ in under 65 year olds to $5 \%$ for over 85 year olds. There was a similarly higher rate of fatal bleeds as age increased [86].It is reasonable to presume that this increased risk with age will be also applicable to the MPN population Patients should receive appropriate counselling regarding the risk of hemorrhage and consideration should be given to the use of proton pump inhibitors which have proven benefit in the reduction of gastrointestinal bleeding associated with aspirin.

Anticoagulation with heparin, oral vitamin K antagonists or NOACs are also associated with a higher risk of hemorrhage in any population. In patients with MPN provoked splanchnic vein thrombosis the sequelae of the initial clot include liver cirrhosis, portal hypertension and oesophageal varices. 
Resulting synthetic liver failure can impact on clotting factor production resulting in an acquired bleeding diathesis. These patients have both a significant rate of hemorrhage and re-thrombosis. [87]. These patients require a multi-disciplinary input from hepatology and hematology with regular screening and treatment of oesophageal varices, careful evaluation of platelet count and coagulation function. An individual risk profile will help to guide the use of an anticoagulant and this should be regularly reviewed with the patient. Guidelines recommend indefinite anticoagulation for SVT in the presence of an MPN where this is possible [45].

\subsection{Special situations}

\subsection{Surgery}

Patients with MPN are at have an increased morbidity and mortality when undergoing surgery. This is associated with thromboembolic events which is perhaps not unexpected. However, hemorrhagic complications also occur with increased frequency which may be related to acquired von Willibrand's disease occurring with elevated platelet counts. A retrospective study in Italian patients with PV and ET found a rate of thrombotic events of $7 \%$ and the rate of major hemorrhage of $10 \%$ after major surgery with an increased risk in those receiving anti-thrombotic prophylaxis [88]. Splenectomy, which may be considered for the management of portal vein thrombosis or transfusion dependent anaemia in MPN is of particular high risk. In one large series of patients undergoing splenectomy with IMF, the operative mortality rate was $9 \%$ and the morbidity rate $31 \%$ [89].

There is uncertainty as to the optimal management of surgery in MPN patients as there is a lack of prospective trials. However, in preparation for surgery, control of erythrocytosis and thrombocytosis should be optimised by venesection for erythrocytosis to ensure the HCT is less than 0.45 . Myelosuppression should be optimised to reduce the platelet count to below $400 \times 10^{9} / \mathrm{I}$. In a low risk patient who routinely does not require myelosuppression consideration should be given to temporary introduction to myelosuppression to control the platelet count for the period of the surgery and the postoperative period. Aspirin should be withheld for 7 days before elective surgery but can be restarted 24 hours after stopping heparin. Low molecular weight heparin at a prophylactic dose is indicated in all patients with MPN undergoing surgery because of the high thrombotic risk. These patients need careful monitoring during and after surgery for both bleeding and thrombotic complications

\subsection{Pregnancy}

The presence of a MPN is a major factor in the prothrombotic condition of pregnancy. In ET, where pregnancies are most common, survey of the literature report success rates (live babies) of $61 \%$ and a first trimester abortion rate of 32\% [90]. However, in ET the presence of a JAK2 mutation was an independent predictor for complications. The live birth rate in this study was $64 \%$ with a relationship between the presence of the JAK2 mutation and fetal loss [91]. Pregnancy and a diagnosis of PV is less common and the literature survey reported a live birth rate in PV of $58 \%$ with a spontaneous first trimester abortion rate of $22 \%$. There are very few reports of pregnancy associated with MF which would be predicted to be associated with a high complication rate. A recent prospective study following pregnancies in MPN, mainly ET in 58 pregnancies (56 singleton and 2 twin pregnancies) resulted in 58 live births. The miscarriage rate was $1.7 / 100$ pregnancies. There were no neonatal deaths but $22 \%$ were below the $10 \%$ centile for growth. This more recent study suggests that MPN pregnancies have better outcomes than perhaps would be anticipated from previous literature [92]. 
Pregnancy in MPN is a high-risk situation and requires specialist management by an obstetrician with experience in the care of high-risk pregnancy and haematologist. If possible, a pre-conception planning meeting should take place to discuss risks and plan management of a pregnancy. The pregnancy should be classified as low or high risk, high risk being where there is any factor in the mother (previous thromboembolic event or hemorrhage related to MPN) or previous pregnancy complication which may have been caused by MPN or a platelet count above $1500 \times 10^{9} / \mathrm{l}$.

All pregnant MPN patients should receive low dose aspirin and prophylactic low molecular weight heparin (LMWH) for 6 weeks postpartum. In PV, the Hct should be reduced with venesection to an $\mathrm{Hct}$ in the middle of the gestation appropriate range. Patients should have frequent appointments with full blood picture monitoring and 6 falling to 4 weekly ultrasound scans. Uterine artery doppler scanning should be carried out at 20 and 24 weeks to see if there is a high resistance index and /or bilateral notching. Persistent bilateral notching is an indication of resistance to flow and possible placental dysfunction.

Cytoreductive therapy with interferon alpha should be considered if the clinical situation indicates such as previous history, events indicating placental dysfunction [93] where it appears to be safe in pregnancy and may help to improve pregnancy outcomes. HU may be less toxic than suspected in human pregnancy but should certainly be avoided at the time of conception and in the first trimester at least [94]. Anagrelide should be avoided in pregnancy as it is a small molecule which can cross the placenta and result in thrombocytopenia in the fetus.

The implications of thromboprophylaxis and mode of delivery must be considered. Many anaesthetists prefer aspirin to be discontinued at least week prior to delivery and dosing of LMWH has to be altered for epidural anaesthesia or caesarean section. In the postpartum period, the risk of thromboembolism is highest hence usually LMWH should be considered for 6 weeks.

\subsection{Cytopenia}

Cytopenias occur in MPN because of the disease or as a side effect of treatment. This is true of anemia which can occur from either the disease process itself or other complication. For instance in ET or PV the development of anemia may be an indication that myelofibrosis is developing. This can occur because of the therapy such as $\mathrm{HU}$ in ET where the $\mathrm{Hb}$ is suppressed as well as the desired reduction in platelet counts the $\mathrm{Hb}$ and other cell counts are reduced below the normal range. In such a case the therapy has to be reduced, or interrupted and then an alternate therapy may need to be considered. Patients with MPN particularly PV can become anemic because of iron deficiency. A patient on a venesection programme will become iron deficient as a result of the treatment but these patients can become iron deficient and clinically anemia as a result of blood loss for other reasons. If the patient is markedly anaemia from iron deficiency then iron replacement can be considered but this must be done with great caution as in PV patient the Hct can rise very rapidly leading to the possibility of catastrophic thromboembolic events. If oral iron is given it should be a low dose with very frequent monitoring of the response.

In MF anaemia can arise as part of the progression of the disorder. Many therapies such as erythropoietin stimulating agents (ESA), thalidomide and anabolic steroids have been used and show some responses. With ruxolitinib which is now the first line agent, there is initially a fall in haemoglobin but then some recovery [24]. Despite the fact that ESAs can activate the JAK/STAT, they have been shown to be safe and may be of some effect in the treatment of anemia MF patients in combination with ruxolitinib [95]. 
Other cytopenias arise because of disease progression particularly in the case of MF and as side effect of treatment where cytoreduction may need to be reduced stopped or alternate therapy initiated. With thrombocytopenia and MF treated with ruxolitinib there are specific dose reductions depending on the platelet count.

\subsection{Expert commentary}

The MPNs are acquired clonal diseases where there a number of complications which can arise leading to considerable morbidity and in some cases mortality. Treatment has been directed a controlling blood counts and prevention of complications. Following the discovery of the driver mutations, research is now directed at the development of predictive scores for prognosis. As these are refined they have the potential to develop into personal predictions which can be used to guide treatment. This leads to the possibility of true personalised medicine where taking into account all information including increasingly detailed molecular diagnostics in the disease management can be guided. The discovery of the driver mutations and the function of the mutated proteins has led to the development of new therapeutic agents which inhibit the JAK/STAT pathway have been introduced which have proved beneficial in the management of many signs and symptoms. There are suggestions that they also reduce the size of the acquired clone size thereby reducing the burden of disease and may led to improved survival.

Weakness in the work carried out so far are that that these are rare diseases and studies have been carried out in large retrospective cohorts. Results are then used to predict prognosis in for future cohorts. While these predictive models have been validated, so many other factors change in cohorts of patients that validity of predictive models has always to be questioned particularly when applied to individual patients. Predictive models need to be validated in prospective cohorts. It is also disappointing that a number of drugs which have been developed and have come to clinical trial have failed in trials and have not therefore come through to licensed therapeutic options.

The ultimate gaol of therapy has to be elimination of the acquired clone and therefore the disease. In doing this it is assumed that removal of the removal of the clone will result in no disease associated events particularly thromboembolic events.

Current management has been directed at treatment of symptoms and prevention of events. However the discovery of the driver mutation clones has led to a different means of treating with the aim of elimination of the clone. More research for better agents is needed and further research into the mechanism of the actions of mutated clones. Even more fundamental questions are also of great relevance. Why do mutated clones arise? What are the factors which lead to this for instance does inflammation play a part in the development of acquired clones?

The main challenge now is to develop safe and effective agents to treat MPNs so that the acquired clone is removed and therefore complications do not arise in other words cure of these acquired diseases. Treatment also needs to be personalised in other words good prognostic information needs to be available so that treatment can be tailored to the individual factors in the patient with a particular disease.

The important issue for patients with MPNs is the wide variety of disabling symptoms from which they suffer. We need better strategies for dealing with or eliminating many of the disabling symptoms which from a patient view may be the main issue rather than cure of the disease.

\subsection{Year view}


With the discovery of the driver mutation and patterns of driver mutations, the field of therapeutics for MPN has altered completely. One inhibitor of the JAK/STAT pathway is now in use in routine practice and has had a major impact on the disease burden. A number of other JAK/STAT pathway inhibitors have entered clinical trials but unfortunately most have not made it to clinical licence and use in practice. However, over the next 5 years new targeted agents are likely to enter trials and hopefully proof safe and effective and enter clinical practice. These agents would be directed at reduction in the size of the acquired clone and ultimately its elimination, in other words potential disease cure. Diagnostics particularly molecular diagnostics will develop rapidly. This with scoring systems for predicting prognosis will be refined and validated in prospective cohorts. These will lead to better predictive systems on which treatment decisions can be based leading to more patient personalised therapeutics. It is likely therefore in 5 years that more refined diagnostics and new therapeutic agents will expand and refine treatment options for MPNs.

\subsection{Key issues}

- Symptoms are common in myeloproliferative Neoplasms and can be assessed with tools such as MPN-SAF TSS

- Fatigue and pruritus can cause major disability

- A variety of therapies may be effective for relief of pruritus

- Ruxolitinib inhibits the JAK/STAT pathway and reliefs symptoms

- Thrombotic events can be treated with anti-platelet agents anticoagulants and cytoreduction

- Hemorrhage is associated with extremely elevated platelet counts

- Surgery is associated with increased risk of events and requires specific management

- Pregnancy is associated with increased events and requires risk assessment and individualised treatment regimens

- Anemia may be a side effect of treatment but may require specific therapy

\section{References}

1. Damashek W. Some speculations on the myeloproliferative syndromes. Blood 1951;6:372-5.

2. **Arber DA, Orazi A, Hasserjian $\mathrm{R}$ et al. The 2016 revision to the World Health Organisation classification of myeloid neoplasms and acute leukemia Blood 2016;127:2391-405.

After 8 years the WHO have revised and refined the classification of malignancy and this paper describes the new classification of myeloid neoplasms including the myeloproliferative neoplasms

3. Baxter EJ, Scott LM, Campbell PJ, et al. Acquired mutation of the tyrosine kinase JAK2 in human myeloproliferative disorders. Lancet 2005; 365: 1054-1061.

4. Nangalia J, Massie CE, Baxter EJ et al. Somatic CALR mutations in Myeloproliferative Neoplasms with non-mutated JAK2. N Eng J Med 2013; 369: 2391-2405

5. Pikman Y, Lee BJ, Mercher T et al. MPLW515L is a novel somatic activating mutation in myelofibrosis with myeloid metaplasia. PLoS 2006;1140-51. 
6. Mesa RA, Schwager S, Radia D et al. The myelofibrosis symptom assessment form (MFSAF): An evidence-based brief inventory to measure quality of life and symptomatic response to treatment in myelofibrosis. Leuk Res 2009;33:1199-1203.

7. Scherber $R$, Dueck $A C$, Johansson $P$ et al. The myeloproliferative neoplasm symptom assessment form (MPN-SAF): International prospective validation and reliability trial in 402 patients. Blood 2011;118:401-8.

8. *Emmanuel RM, Dueck AC, Geyer HL et al. Myeloproliferative neoplasm (MPN) symptom assessment form total symptom score: prospective international assessment of an abbreviated symptom burden scoring system among patients with MPNs. J Clin Oncol 2012;20:4098-103.

Symptom assessment form MPN-SAF TSS is described and tested which has widespread utility.

9. *Anderson LA, James G, Duncombe AS, et al. Myeloproliferative neoplasm patient symptom burden and quality of life: Evidence of significant impairment compared to controls. Am J Hematol 2015;90:864-70.

Symptoms in patients with all types of myeloproliferative neoplasms are more than in controls

10. Mesa RA, Niblack J, Wadleigh $M$ et al. The burden of fatigue and quality of life in myeloproliferative disorders(MPDs). Cancer 2006;109:68-76.

11. Huberty J, Eckert R, Gowin K et al.Feasibitlity of online yoga for symptom management in patients with myeloproliferative neoplasms. Haematologica2017;102e384-8.

12. Gilbert HS, Warner RR, Wasserman LR. A study of histamine in myeloproliferative disease. Blood 1966;28:795-806.

13. Pieri, Bogani C, Gugliemelli P et al. The JAK2V617 mutation induces constitutive activation and agonist hypersensitivity in basophils from patients with polycythemia. Haematologica 2009;94:1537-45.

14. Weick JK, Donovan PB, Najean Y et al. The use of cimetidine for the treatment of pruritus in polycythemia vera. Arch Intern Med 1982;142:241-2.

15. Diehn F,Tefferi A. Pruritus in polycythaemia vera: prevalence, laboratory correlated and management. Br J Haematol 2001;115:619-21.

16. Tefferi A, Fonesca R. Selective serotonin reuptake inhibitors are effective in the treatment of polycythemia vera-associated pruritus. Blood 2002;99:2627.

17. Jeanmougin M, Rain JD, Najean Y. Efficacy of photochemotherapy on severe pruritus in polycythemia vera. Ann Hematol 1996;73:91-3.

18. Hernandez-Nunez A, Dauden E, Cordoba S et al. Water-induced pruritus in haematologically controlled polycythaemia vera: response to phototherapy. J Dermatol Treat 2001;12:107-9.

19. Menage HD, Norris PG, Hawk JL et al. The efficacy of psoralen photochemotherapy in the treatment of aquagenic pruritus. Br J Dermatol 1993;129:163-5.

20. Baldo A, Sammarco E, Monfrecola M.UVB phototherapy for pruritus in polycythaemia vera. Dermatol Treat 1996; 7:245-6.

21. Baldo A, Sammarco E, Plaitano R et al. Narrowband (TL-01) ultraviolet B phototherapy for pruritus in polycythaemia vera. Br J Bermatol 2002;147:979-81.

22. Finelli $C$, GugliottaL, Gamberi B et al. Relief of intractable pruritus in polycythemia vera with recombinant interferon alfa. Am J Hematol 1993;43:316-8. 
23. Muller EW, de Wolf JT, Egger R, et al. Long-term treatment with interferon-alpha $2 b$ for severe pruritus in patients with polycythaemia vera. $\mathrm{Br} J$ Haematol 1995;89:313-8.

24. **Verstovsek S, Mesa RA, Gotlib J et al. A double -blind, placebo -controlled trial of ruxolitinib for myelofibrosis N Eng J Med 2012; 366:799-087.

COMFORT-1 trial, the first phase 3 showing the efficacy of ruxolitinib in the treatment of myelofibrosis.

25. **Vannucchi AM, Kiladjian J-J, Greisshammer $\mathrm{M}$ et al. Ruxolitinib versus standard therapy for the treatment of polycythemia vera. N Eng J Med 2015; 372:42635.

Phase 3 trial showing efficacy of ruxolitinib in treatment of polycythemia vera.

26. Geyer H, Mesa RA. Assessing disease burden in patients with classis MPNs. Best Pract Res Clin Haematol 2014; 27: 107-19.

27. Barosi G, Ambrosetti A, Centra A et al. Splenectomy and risk of blast transformation in myelofibrosis with myeloid transformation. Blood 1998; 33630-6.

28. Aruch D, Schwartz M, Mascarenhas J et al. Continued role of splenectomy in the management of myelofibrosis. Clinical Lymphoma Myeloma and Leukemia 2016; e133-7.

29. Kitanaka A, Takenaka K Shide K et al. Splenic irradiation provides transient palliation for symptomatic splenomegaly associated with primary myelofibrosis: a report on 14 patients. Int J Hematol 2016;103: 423-8.

30. Gruppo Italiano Studio Policitemia. Polycythemia vera: the natural history of 1213 patients followed for 20 years. Ann Intern Med 1995; 123:656-664.

31. Landolfi R, Di Gennaro L, Barbui T et al. Leukocytosis as a major thrombotic risk factor in patients with polycythemia vera. Blood 2007 109:2446-2452.

32. Barbui $T$, Carobbio $A$, Rumi $E$ et al. In contemporary patients with polycythemia vera, rates of thrombosis and risk factors delineate a new clinical epidemiology, Blood 2014; 124:3021-3.

33. Marchioli R, Finazzi G, Landolfi R, et al. Vascular and neoplastic risk in a large cohort of patients with polycythemia vera. J Clin Oncol 2005; 23:2224-32.

34. Harrison CN, Campbell PJ, Buck G et al. Hydroxyurea compared with anagrelide in high-risk essential thrombocythemia N Engl J Med. 2005 ;353:33-45

35. Barbui T, Finazzi G, Carobbio A, et al. Development and validation of an International Prognostic Score of thrombosis in World Health Organization-essential thrombocythemia (IPSET-thrombosis), Blood 2012; 120:5128-33.

36. Rumi E, Pietra D, Ferretti V, et al. JAK2 or CALR mutation status defines subtypes of essential thrombocythemia with substantially different clinical course and outcomes. Blood 2014; 123: 1544-51.

37. Tefferi A, Barbui T. Polycythemia vera and essential thrombocythemia: 2015 update on diagnosis, risk-stratification and management. Am J Hematol. 2015;90:162-73.

38. Falchi L, Kantarjiaz HM,-Verstovsek S. Assessing the thrombotic risk of patients with essential thrombocythemia in the genomic era. Leukemia 2017;31:1845-54.

39. Barbui T, Falanga AM. Molecular biomarkers of thrombosis in myeloproliferative neoplasms Thrombosis Research 2016; 140 , S71 -5.

40. Barbui T, Thiele J, Carobbio A. Disease characteristics and clinical outcome in young adults with essential thrombocythemia versus early/prefibrotic primary myelofibrosis. Blood 2012; 120:56971.

41. Vannucchi AM, Barbui T, Cervantes F et al. Philadelphia Chromosome-Negative Chronic Myeloproliferative Neoplasms; ESMO Clinical Practice Guidelines. Ann Oncol 2015; 26 (suppl 5): v85-v99. 
42. Harrison $\mathrm{CN}$, Bareford $\mathrm{D}$, Butt $\mathrm{N}$ et al. Guideline for investigation and management of adults and children presenting with a thrombocytosis, Br J Haematol 2010;149:352-75.

43. Smalberg JH, Arends LR, Valla DC. Myeloproliferative neoplasms in Budd-Chiari syndrome and portal vein thrombosis: a meta-analysis. Blood 2012; 120:4921-8.

44. Plompen EP, Valk PJ, Chul. Somatic Calreticulin Mutations In Patients With Budd-Chiari Syndrome And Portal Vein Thrombosis. Haematologica June 2015 100: e226-8.

45. Tait $\mathrm{C}$, Baglin $\mathrm{T}$, Watson $\mathrm{H}$, et al, Guidelines on the investigation and management of venous thrombosis at unusual sites. Br J Haematol 2012;159:28-38.

46. Colaizzo D, Amitrano L, Guardascione MA. Outcome of patients with splanchnic venous thrombosis presenting without overt MPN: a role for the JAK2 V617F mutation reevaluation. Thromb Res 2013; 132:e99-104.

47. Thatipelli MR, McBane RD, Hodge DO et al, Survival and recurrence in patients with splanchnic vein thromboses. Clin Gastroenterol Hepatol 2010;8:200-5.

48. Smalberg JH, Koehler E, Darwish Murad S et al. The JAK2 46/1 haplotype in Budd-Chiari syndrome and portal vein thrombosis. Blood. 2011;117:3968-73.

49. Barbui T, Masciulli A, Marfisi MR, et al. White blood cell counts and thrombosis in polycythemia vera: a subanalysis of the CYTO-PV study. Blood 2015; 126:560-1.

50. Carobbio A, Finazzi G, Guerini V,et al. Leukocytosis is a risk factor for thrombosis in essential thrombocythemia: interaction with treatment, standard risk factors, and Jak2 mutation status. Blood 2007; 109:2310-3.

51. Wolanskyj AP, Schwager SM, McClure RF et al. Essential thrombocythemia beyond the first decade: life expectancy, long-term complication rates, and prognostic factors, Mayo Clin Proc. 2006;81:159-66

52. Passamonti F, Thiele J, Girodon F et al. A prognostic model to predict survival in 867 World Health Organization-defined essential thrombocythemia at diagnosis: a study by the International Working Group on Myelofibrosis Research and Treatment. Blood 2012; 120:1197-201.

53. De Grandis M, Cambot M, Wautier M, et al. JAK2V617F activates Lu/BCAM-mediated red cell adhesion in polycythemia vera through an EpoR-independent Rap1/Akt pathway, Blood 2013; 121:658-65.

54. Landolfi R, Marchioli R, Kutti J et al, Efficacy and safety of low-dose aspirin in polycythemia vera, N Engl J Med. 2004;350:114-24.

55. Zahavi J, Zahavi M, Firsteter E et al. An abnormal pattern of multiple platelet function abnormalities and increased thromboxane generation in patients with primary thrombocytosis and thrombotic complications. Eur J Haematol 1991;47:326-32.

56. Arellano-Rodrigo E, Alvarez-Larran A, Reverter JC et al. Increased Platelet And Leukocyte Activation As Contributing Mechanisms For Thrombosis In Essential Thrombocythemia And Correlation With The JAK2 Mutational Status. Haematologica 2006; 91: 169-75.

57. Falanga $A$, Marchetti $M$, Vignoli A et al. Leukocyte-platelet interaction in patients with essential thrombocythemia and polycythemia vera. Exp Hematol 2005;33:523-30.

58. Lussana F, RambaldiA. Inflammation and myeloproliferative neoplasms. J Autoimmun. 2017; pii: S0896-8411(17)30430-4.

59. Ridker PM, Everett, Thuren T et al. Antiinflammatory Therapy with Canakinumab for Atherosclerotic Disease. N Engl J Med 2017;377:1119-31.

60. Barbui T, Carobbio A, Guido Finazzi et al. Inflammation And Thrombosis In Essential Thrombocythemia And Polycythemia Vera: Different Role Of C-Reactive Protein And Pentraxin 3. Haematologica 2011; 96:315-318.

61. Barbui T, Masciulli A, Ghirardi A. ACE inhibitors and cytoreductive therapy in polycythemia vera, Blood 2017;129:1226-7.

62. Jaiswal S, Natarajan P, Silver AJ et al. Clonal hematopoiesis and risk of atherosclerotic cardiovascular disease. N Engl J Med. 2017;377:111-21. 
63. McMullin MF, Bareford D, Campbell $P$ et al., Guidelines for the diagnosis, investigation and management of polycythaemia/erythrocytosis. Br J Haematol 2005;130:174-95.

64. Chu D, Hillis CM, Leong DP et al. Benefits and risks of antithrombotic therapy in essential thrombocythemia Ann Intern Med 2017;167:170-80.

65. Alvarez-Larrán A, Cervantes F, Pereira A et al. Observation versus antiplatelet therapy as primary prophylaxis for thrombosis in low-risk essential thrombocythemia. Blood 2010; 116:1205-10.

66. Marchioli R, Finazzi G, Specchia G et al. The CYTO-PV: A Large-Scale Trial Testing the Intensity of CYTOreductive Therapy to Prevent Cardiovascular Events in Patients with Polycythemia Vera. Thrombosis 2011;2011:794240

67. Barbui T, Barosi G, Birgegard G et al. Philadelphia-Negative Classical Myeloproliferative Neoplasms: Critical Concepts and Management Recommendations From European LeukemiaNet. J Clin Oncol. 2011;29:761-70.

68. Barbui T, Vannucchi AM, Finazzi G. A reappraisal of the benefit-risk profile of hydroxyurea in polycythemia vera: A propensity-matched study. Am J Hematol. 2017; Jul 11

69. Björkholm M, Derolf AR, Hultcrantz $M$ et al. Treatment-related risk factors for transformation to acute myeloid leukemia and myelodysplastic syndromes in myeloproliferative neoplasms. J Clin Oncol. 2011;29:2410-5.

70. Gisslinger H, Gotic M, Holowiecki J et al. Anagrelide compared with hydroxyurea in WHOclassified essential thrombocythemia: the ANAHYDRET Study, a randomized controlled trial. Blood 2013;121:1720-8.

71. Birgegård G. The Use of Anagrelide in Myeloproliferative Neoplasms, with Focus on Essential Thrombocythemia. Curr Hematol Malig Rep. 2016; 11:348-355.

72. Kiladjian JJ, Cassinat B, Chevret $\mathrm{S}$ et al. Pegylated interferon-alfa-2a induces complete hematologic and molecular responses with low toxicity in polycythemia vera, Blood 2008; 112:3065-72.

73. Quintás-Cardama A, Abdel-Wahab O, Manshouri T, et al. Molecular analysis of patients with polycythemia vera or essential thrombocythemia receiving pegylated interferon $\alpha$-2a Blood 2013;122:893-901.

74. Boddu P, Falchi L Hosing C et al. The role of thrombocytapheresis in the contemporary management of hyperthrombocytosis in myeloproliferative neoplasms: a case-based review. Leukemia Research 2017;58: 14-22.

75. Harrison CN, Vannucchi AM, Kiladjian JJ, et al. Long-term findings from COMFORT-II, a phase 3 study of ruxolitinib vs best available therapy for myelofibrosis. Leukemia 2016; 30:1701-7.

76. Pieri L, Pancrazzi A, Pacilli A, et al. JAK2V617F complete molecular remission in polycythemia vera/essential thrombocythemia patients treated with ruxolitinib. Blood 2015; 125:3352-3.

77. Vannucchi AM, Verstovsek S, Guglielmelli P et al. Ruxolitinib reduces JAK2 p.V617F allele burden in patients with polycythemia vera enrolled in the RESPONSE study. Ann Hematol 2017;96:1113-20.

78. Cruzado J, Rico J, Grinyó J. The renin angiotensin system blockade in kidney transplantation: pros and cons Transpl Int 2008;21:304-13.

79. Patel MR, Mahaffey KW, Garg J et al. Rivaroxaban versus warfarin in nonvalvular atrial fibrillation. N Engl J Med. 2011;365:883-91.

80. Cohen AT,Hamilton M, Mitchell SA et al. Comparison of the Novel Oral Anticoagulants Apixaban, Dabigatran, Edoxaban, and Rivaroxaban in the Initial and Long-Term Treatment and Prevention of Venous Thromboembolism: Systematic Review and Network MetaAnalysis, PLoS One. 2015;10:e0144856

81. lanotto JC, Couturier MA, Galinat $\mathrm{H}$, Administration of direct oral anticoagulants in patients with myeloproliferative neoplasms. Int J Hematol. 2017 Jun 16. 
82. Ellis $\mathrm{MH}$, Lavi $\mathrm{N}$, Vannucchi $\mathrm{A}$ et al. Treatment of thromboembolic events coincident with the diagnosis of myeloproliferative neoplasms: A physician survey. Thrombosis Research 2014;134: 251-4.

83. Kaifie $A$, Kirschner $M$, Wolf $D$ et al. Bleeding, thrombosis, and anticoagulation in myeloproliferative neoplasms (MPN): analysis from the German SAL-MPN-registry J Hematol Oncol. 2016;9:18.

84. Budde U, van Genderen PJ et al. Acquired von Willebrand disease in patients with high platelet counts. Semin Thromb Hemost. 1997;23:425-31.

85. Rumi E, Cazzola M. How we treat essential thrombocythemia Blood 2016;128:2403-14.

86. Li L, Geraghty OC, Mehta Z et al. Age-specific risks, severity, time course, and outcome of bleeding on long-term antiplatelet treatment after vascular events: a population-based cohort study Lancet. 2017;390:490-499.

87. Sekhar M, McVinnie K, Burroughs AK et al. Splanchnic vein thrombosis in myeloproliferative neoplasms. Br J Haematol 2013;162:730-47

88. Ruggeri M, Rodeghiero F, Tosetto A et al. Postsurgery outcomes in patients with polycythemia vera and essential thrombocythemia: a retrospective survey. Blood 2008;111:666-71.

89. Tefferi A, Mesa RA, Nagomey DM et al. Splenectomy in myelofibrosis with myeloid metaplasia: a single-institution experience with 223 patients. Blood 2000;95:2226-33.

90. Griesshammer M, Struve S, Barbui T. Management of Philadelphia negative chronic myeloproliferative disorders in pregnancy. Blood Reviews 2008; 22:235-45.

91. Passamonti F, Randi ML, Rumi E et al. Increased risk of pregnancy in patients with essential thrombocythemia carrying the JAK2 (617V >F) mutation. Blood 2007;110:485-9.

92. *Alimam S, Bewley S, Chappell LC et al. Pregnancy outcomes in myeloproliferative neoplasms: UK prospective cohort study. Br J Haematol 2016;175:31-6.

Prospective study of maternal and fetal outcomes in myeloproliferative pregnancy.

93. Martinelli P, Martinelli V, Agangi A et al. Interferon alfa treatment for pregnant women affected by essential thrombocythemia: Case reports and a review. Am J Obstet Gyn. 2004;191:2016-20.

94. Harrison C. Pregnancy and its management in the Philadelphia negative myeloproliferative diseases. Br J Haematol 2005;129:293-306.

95. McMullin MF Harrison CN, Niederwiesser D et al. The use of erythropoiesis-stimulating agents with ruxolitinib in patients with myelofibrosis in COMFORT-II: an open-label, phase 3 study assessing efficacy and safety of ruxolitinib versus best available therapy in the treatment of myelofibrosis. Exp Hematol Oncol 2015; 4: 26.

96. Barbui T, Vannucchi, AM, Buxhofer-Ausch V et al. Practice-relevant revision os IPSETthrombosisbased on 1019 parients with WHO-defined essential thrombocythemia. Blood Cancer Journal 2015;5: e369. 
Financial disclosures

MFM has been in the speaker's bureau and advisory boards for Novartis, and advisory board for Gilead. 